\title{
Thermal phase transitions of dimensionally continued AdS black holes
}

\author{
Xiao-Mei Kuang * and Olivera Miskovic ${ }^{\dagger}$ \\ Instituto de Física, Pontificia Universidad Católica de Valparaíso, \\ Casilla 4059, Valparaíso, Chile
}

February 3, 2017

\begin{abstract}
We study the thermal phase transitions of charged black holes in dimensionally continued gravity in anti-de Sitter space. We find the van der Waals-like phase transition in the temperature-entropy plane of the black holes with spherical horizons in even dimensions, and there is no such phase transition of the black holes with flat and hyperbolic geometries. Near the critical inflection point, the critical exponent is computed and its value does not depend on the dimension. The Maxwell equal area law is checked to be fulfilled in the temperature-entropy diagram for the first order phase transition. In odd dimensions, there are no thermal phase transitions for the black hole with any geometry of the horizon.
\end{abstract}

\section{Introduction}

The black hole thermodynamics is a challenging topic in black hole physics that has been studied for decades because of its non-perturbative, quantum nature. The phase diagrams of black holes exhibit rich structures that carry information about stability of these systems and, for that reason, have been attracting more attention and effort with time. Among them, the van der Waals-like behavior of black holes has been widely studied because of its striking similarity to the liquid-gas phase transition in the ordinary thermodynamic systems.

\footnotetext{
*xmeikuang@gmail.com

†olivera.miskovic@pucv.cl
} 
The earliest analysis of first order phase transitions in black hole systems can be found in refs. [1, 2], where the authors studied the inverse temperature-horizon diagram. It was found that, as the charge of black hole decreases to a critical value, the system undergoes a second-order phase transition, and below the critical charge, the black hole always presents first-order phase transition. This process is very similar to the one of the van der Waals liquid-gas transition. Later, by treating the negative cosmological constant as the pressure and the physical volume enclosed by the black hole horizon as its thermodynamical conjugated quantity, and identifying the black hole mass as enthalpy instead of internal energy, the black hole thermodynamic is re-studied in the extended phase space that includes the pressure and volume [3, 4, [5, 6], and the first law agrees with the corresponding Smarr relation [7, 8, 9]. Further, first attempts to study the phase structure in the pressure-volume, $P-V$, diagram was made for the charged ReissnerNördstrom (RN) anti-de Sitter (AdS) black hole with fixed charge and the van der Waalslike phase transition was observed in [10]. Namely, as the temperature increases, the black hole undergoes the first-order phase transition and then the second-order phase transition at the critical point, until it arrives at a stable phase. This proposal led to many generalizations in more general cases [11, 12, 13, 14, 15, 16]. In particular, in Ref. [16], the $P V$ criticality was discussed in a general framework, based on the Smarr formula and the first law of thermodynamics, revealing its universality.

In this work, we are interested in the phase structure of charged black holes in dimensionally continued gravity proposed in [17]. It is a higher-dimensional Lovelock AdS theory which possesses a unique AdS vacuum [18], so that the only parameters in the theory are the gravitational constant, $\kappa$, and the AdS radius, $\ell$. In particular, this theory becomes Chern-Simons AdS gravity in odd dimensions [19] and Born-Infeld AdS gravity in even dimensions. The theory possesses topological black hole solutions whose properties and thermodynamics were studied in [17, 20, 21]. A full boundary series of counterterms that renders dimensionally continued gravity finite in the IR region, is presented in Ref.[22].

In this work we will focus on thermal phase transitions in the temperature-entropy, $T$ $S$, plane in ordinary black hole phase space, rather than in the $P-V$ plane in the extended space, for the following two reasons. On one hand, it was argued in [23] that these two behaviors are dual to each other because both of them stem from the same equation, i.e., the expression for the Hawking temperature of the black hole. However, working in one side of the duality might be significantly simpler. For example, the Maxwell's equal area law for charged AdS black holes was possible to solve exactly in the $T$ - $S$ plane, whereas the solution was found only perturbatively around the critical values in the P-V plane [23. The van der Waals-like phase transition in the $T$-S plane was also observed in [24] and the references therein.

On the other hand, differently than in general relativity, the entropy of dimension- 
ally continued black holes depends on both the horizon $r_{+}$and the cosmological constant through $\ell$. This leads to a highly non-linear expression for the pressure, making it technically very difficult to study phase diagrams in the extended phase space of such non-linear objects. In fact, it is much more convenient to analyze the phase structure in the $T$ - $S$ plane, where the parameter $\ell$ does not vary, expecting that its phase structure is similar to the one in the $P-V$ plane due to the duality.

Let us also comment that, as nicely explained in a footnote of Ref. [23], this conjectured duality of descriptions in two different planes is analogous to the T-duality of string theory, where the spectrum of a closed string wrapped around a compact dimension of some radius $R$ can be seen as the spectrum of a closed string wrapped around a compact dimension of the inverse radius, $1 / R$. The coupling constants in the corresponding dual theories are inverse to each other. Similarly, in black hole thermodynamics, the state equation can be understood either as the Hawking temperature $T$ for the black hole in a charged background, or as the pressure $P$ of a van der Waals fluid enclosed in a (specific) volume $V$ and heated at the temperature $T$. Considering the former treatment means to focus on the $T-S$ phase space, while the latter treatment means to focus on the $P-V$ phase space. Exchanging the roles of $P$ and $T$ is analogous to exchanging Kaluza-Klein momentum and winding modes in string theory. An inversion of a coupling constant due to the duality in black hole thermodynamics refers to the Boltzman constant $\left(k_{\mathrm{B}} \rightarrow 1 / k_{\mathrm{B}}\right)$.

In Dimensionally Continued Gravity, we find that the van der Waals-like phase transition only occurs in black holes with spherical horizons in Born-Infeld AdS gravity in even dimensions, and there are no such phase transitions in Chern-Simons AdS gravity in odd dimensions. For the black holes that exhibit a van der Waals-like behavior, our results show that the critical exponent near the critical point is consistent with that found in the mean field theory and the Maxwell equal area law is satisfied for the first order phase transition.

The rest of this paper is organized as follows. In Section 2, we briefly review dimensionally continued gravity and the charged, topological black holes. We discuss the existence of thermal phase transitions and their properties, such as critical exponents and the Maxwell equal area law, in even dimensions in Section 3 and odd dimensions in Section 4, respectively. Section 5 contains our conclusions and discussion.

\section{Charged dimensionally continued black holes}

Lovelock gravity action is a linear combination of dimensionally continued Euler densities [25, 26],

$$
I_{G}=\kappa \int d^{D} x \sqrt{-g} \sum_{p=0}^{n-1} \frac{\alpha_{p}}{2^{p}} \delta_{\nu_{1} \cdots \nu_{2 p}}^{\mu_{1} \cdots \mu_{2 p}} R_{\mu_{1} \mu_{2}}^{\nu_{1} \nu_{2}} \cdots R_{\mu_{2 p-1} \mu_{2 p}}^{\nu_{2 p-1} \nu_{2 p}}
$$


where an integer $n$ is related to the space-time dimension, so that $D=2 n$ in even dimensions and $D=2 n-1$ in odd dimensions, and $\kappa$ describes the strength of gravitational interaction. Here, $\delta_{\nu_{1} \cdots \nu_{2 p}}^{\mu_{1} \cdots \mu_{2 p}}=\operatorname{det}\left[\delta_{\nu_{1}}^{\mu_{1}} \delta_{\nu_{2}}^{\mu_{2}} \cdots \delta_{\nu_{2 p}}^{\mu_{2 p}}\right]$ is the completely antisymmetric product of $2 p$ Kronecker's deltas. The gravitational action (2.1) is the most general one leading to the second order field equations in the metric field $g_{\mu \nu}(x)$. In a special case of dimensionally continued gravity (DCG) [17], the coefficients $\alpha_{p}$ are fixed so that the vacuum solution corresponds to the constant curvature space, $R_{\alpha \beta}^{\mu \nu}=-\frac{1}{\ell^{2}} \delta_{\alpha \beta}^{\mu \nu}$, with the AdS radius $\ell$. In the notation of Ref.[18], the coefficients are 1 ]

$$
\alpha_{p}=-\left(\begin{array}{c}
n-1 \\
p
\end{array}\right) \frac{(D-2 p-1) !}{\ell^{2(n-p-1)}} .
$$

From (2.1) and (2.2), it is clear that the only parameters in the theory are $\kappa$ and $\ell$. To obtain charged solutions, one has to couple gravity to the electromagnetic field,

$$
I=I_{G}-\frac{1}{4 e^{2}} \int d^{D} x \sqrt{-g} F^{2}
$$

with $e^{2}$ controlling the strength of electromagnetic interaction. Equations of motion obtained from the action principle are the Maxwell equation,

$$
\nabla_{\nu} F^{\mu \nu}=0
$$

and the gravitational equation for DCG,

$$
-\kappa \sum_{p=0}^{n-1} \frac{\alpha_{p}}{2^{p-1}} \delta_{\mu \mu_{1} \cdots \mu_{2 p}}^{\nu \nu_{1} \cdots \nu_{2 p}} R_{\nu_{1} \nu_{2}}^{\mu_{1} \mu_{2}} \cdots R_{\nu_{2 p-1} \nu_{2 p}}^{\mu_{2 p-1} \mu_{2 p}}=\frac{1}{e^{2}}\left(\frac{1}{2} \delta_{\mu}^{\nu} F^{2}-2 F_{\mu \lambda} F^{\nu \lambda}\right)
$$

or equivalently

$$
\begin{gathered}
-\frac{\kappa}{2^{n-2}} \delta_{\mu \mu_{2} \cdots \mu_{2 n-1}}^{\nu \nu_{2} \cdots \nu_{2 n-1}}\left(R_{\nu_{2} \nu_{3}}^{\mu_{2} \mu_{3}}+\frac{1}{\ell^{2}} \delta_{\nu_{2} \nu_{3}}^{\mu_{2} \mu_{3}}\right) \cdots\left(R_{\nu_{2 n-2} \nu_{2 n-1}}^{\mu_{2 n-2} \mu_{2 n-1}}+\frac{1}{\ell^{2}} \delta_{\nu_{2 n-2} \nu_{2 n-1}}^{\mu_{2 n-2} \mu_{2 n-1}}\right) \\
=\frac{1}{e^{2}}\left(\frac{1}{2} \delta_{\mu}^{\nu} F^{2}-2 F_{\mu \lambda} F^{\nu \lambda}\right) .
\end{gathered}
$$

From the above form of the gravitational equation it is clear that, in absence of the matter fields, the equation $\left(R+\frac{1}{\ell^{2}} \delta^{[2]}\right)^{n-1}=0$ yields, as a particular solution, a maximally symmetric space with the unique AdS radius $\ell$.

The field equations (2.4) and (2.5) possess a static, spherically symmetric solution. The electromagnetic potential $A_{\mu}=\phi(r) \delta_{\mu}^{t}$ reads

$$
\phi(r)=\phi_{\infty}-\frac{Q}{(D-3) r^{D-3}},
$$

\footnotetext{
${ }^{1}$ In this reference, the action $I_{G}=\kappa \int \sum_{p=0}^{n-1} \tilde{\alpha}_{p} L_{p}$ is written in terms of differential forms $L_{p}=$ $\epsilon_{a_{1} \ldots a_{D}} R^{a_{1} a_{2}} \cdots R^{a_{2 p-1} a_{2 p}} e^{a_{2 p+1}} \cdots e^{a_{D}}$. The coefficients $\tilde{\alpha}_{p}$ are related to $\alpha_{p}$ as $\alpha_{p}=-\tilde{\alpha}_{p}(D-2 p) !$.
} 
where $Q$ is an electric charge of the black hole, with the electric field $F_{t r}=Q / r^{D-4}$. The metric describes topological black holes,

$$
d s^{2}=-f^{2}(r) d t^{2}+\frac{d r^{2}}{f^{2}(r)}+r^{2} d \Sigma_{D-2}^{2},
$$

where $d \Sigma_{D-2}^{2}=\gamma_{m n}(y) d y^{m} d y^{n}$ is a constant curvature transversal section with spherical $(k=1)$, hyperbolic $(k=-1)$ or planar $(k=0)$ geometry, whose area is $\Omega_{D-2}$. First integral of the equations of motion, with the integration constant $\mu$, is common for all Lovelock gravities,

$$
\sum_{p=0}^{n-1} \frac{\alpha_{p}}{(D-2 p-1) !}\left(\frac{k-f^{2}}{r^{2}}\right)^{p}=\frac{\mu}{r^{D-3}}-\frac{Q^{2}}{(D-3) \kappa e^{2} r^{2 D-6}},
$$

and, in the DCG case, it becomes

$$
\left(\frac{1}{\ell^{2}}+\frac{k-f^{2}}{r^{2}}\right)^{n-1}=\frac{\mu}{r^{D-1}}-\frac{Q^{2}}{(D-3) \kappa e^{2} r^{2 D-4}} .
$$

It is convenient to normalize the gravitational and electromagnetic constants as

$$
\kappa=\frac{1}{\Omega_{D-2} G}, \quad e^{2}=\Omega_{D-2} .
$$

Then the mass of the black hole $M$ and the integration constant $\mu$ are related by [18]

$$
\mu=2 G M+\delta_{D, 2 n-1},
$$

where the additive constant is chosen so that the black hole horizon shrinks to a point for $M \rightarrow 0$, and is non-vanishing only in Chern-Simons gravity. The general solution becomes [17, 20]

$$
f^{2}(r)=k+\frac{r^{2}}{\ell^{2}}-\left(\frac{2 G M+\delta_{D, 2 n-1}}{r^{D-2 n+1}}-\frac{G Q^{2}}{(D-3) r^{2(D-n-1)}}\right)^{\frac{1}{n-1}} .
$$

In what follows, we will also set $G=1$.

\section{Even-dimensional charged black holes and thermal phase transitions}

In even dimensions $D=2 n$, the spacetime of a charged topological black hole in DCG is described by the metric (2.8) with the metric function (2.13),

$$
f^{2}(r)=k+\frac{r^{2}}{\ell^{2}}-\left[\frac{2 M}{r}-\frac{Q^{2}}{(D-3) r^{D-2}}\right]^{\frac{1}{n-1}} .
$$


For $n=2$, the solution recovers the four-dimensional RN AdS black hole.

The equation of the horizon, $f^{2}\left(r_{+}\right)=0$, enables to express the black hole mass $M$ in terms of the horizon $r_{+}$as

$$
M=\frac{r_{+}}{2}\left[\left(k+\frac{r_{+}^{2}}{\ell^{2}}\right)^{n-1}+\frac{Q^{2}}{(D-3) r_{+}^{D-2}}\right] .
$$

The black hole temperature $T$ is calculated in a standard way, from the Euclidean continuation of the spacetime, where the Euclidean period $T^{-1}$ avoids a conical singularity near the horizon if $T=\left.\frac{1}{4 \pi} \partial_{r} f^{2}\right|_{r_{+}}$. Then the metric (3.1) gives

$$
T=\frac{1}{4 \pi(n-1) r_{+}}\left[k+(2 n-1) \frac{r_{+}^{2}}{\ell^{2}}-\frac{Q^{2}}{r_{+}^{D-2}}\left(k+\frac{r_{+}^{2}}{\ell^{2}}\right)^{2-n}\right],
$$

and the entropy has the form [20]

$$
S=\int_{0}^{r_{+}} d r_{+} \frac{1}{T}\left(\frac{\partial M}{\partial r_{+}}\right)_{Q}=\pi \ell^{2}\left[\left(k+\frac{r_{+}^{2}}{\ell^{2}}\right)^{n-1}-k\right] .
$$

Thermal properties of these black holes have been discussed in [20, 21]. In these papers, the authors studied critical behavior of DCG black holes by identifying the divergencies of thermal capacity, but no nature of these transitions was discussed. They found that phase transitions are possible only in even dimensions.

In the current study, we are interested in the van der Waals-like phase transitions in the $T-S$ plane. Namely, the entopy (3.4) is a function of the horizon, thus from the state equation (3.3), the temperature - which is a function of the horizon $r_{+}$and the charge $Q^{-}$ can be seen as a function of the entropy and the charge, $T=T(S, Q)$. The $T$ - $S$ diagram corresponds to a curve in the $T-S$ plane when the charge is kept fixed.

First we focus on the case with compact horizon $(k=1)$ and work in the ensemble with the fixed charge $Q$.

Combining (3.3) and (3.4), we eliminate $r_{+}$from the equations and obtain the temperature $T$ in terms of the entropy $S$ expressed via the variable $s=\frac{S}{\pi \ell^{2}}+1$,

$$
T=\frac{-\ell^{2-2 n} Q^{2} s^{\frac{2-n}{n-1}}+\left((2 n-1) s^{\frac{1}{n-1}}-2 n+2\right)\left(s^{\frac{1}{n-1}}-1\right)^{n-1}}{4 \pi \ell(n-1)\left(s^{\frac{1}{n-1}}-1\right)^{n-\frac{1}{2}}} .
$$


In first few even dimensions, the state equation $T(S, Q)$ reads

$$
\begin{aligned}
& n=2: \quad T=\frac{-Q^{2}+3 \ell^{2} s^{2}-5 \ell^{2} s+2 \ell^{2}}{4 \pi \ell^{3}(s-1)^{3 / 2}}, \\
& n=3: \quad T=\frac{-Q^{2}+5 \ell^{4} s^{2}-14 \ell^{4} s^{3 / 2}+13 \ell^{4} s-4 \ell^{4} \sqrt{s}}{8 \pi \ell^{5} \sqrt{s}(\sqrt{s}-1)^{5 / 2}}, \\
& n=4: \quad T=\frac{-Q^{2}+7 \ell^{6} s^{2}-27 \ell^{6} s^{5 / 3}+39 \ell^{6} s^{4 / 3}-25 \ell^{6} s+6 \ell^{6} s^{2 / 3}}{12 \pi \ell^{7} s^{2 / 3}\left(s^{1 / 3}-1\right)^{7 / 2}} .
\end{aligned}
$$

Similarly as in case of the van-der Waals $P-V$ diagram, the critical point $\left(S_{c}, Q_{c}, T_{c}\right)$ in the $T$-S plane is obtained as the inflection point of the curve $T(S)$ for constant $Q_{c}$ that satisfies the conditions

$$
\left.\frac{\partial T}{\partial S}\right|_{Q}=0,\left.\quad \frac{\partial^{2} T}{\partial S^{2}}\right|_{Q}=0 .
$$

Requiring that first derivative of (3.5) with respect to the entropy (or the variable $s$ ) vanishes, we find the electric charge as a function of entropy,

$$
Q_{c}^{2}=-\frac{\ell^{2 n-2}(2 n-1) s^{\frac{1}{n-1}}-2 n}{(4 n-5) s^{\frac{1}{n-1}}-2 n+4} s\left(s^{\frac{1}{n-1}}-1\right)^{n-1}
$$

from where the temperature for critical $Q_{c}$ becomes

$$
T_{c}=\frac{2(2 n-1) s^{\frac{2}{n-1}}-(6 n-7) s^{\frac{1}{n-1}}+2(n-2)}{2 \pi \ell\left(s^{\frac{1}{n-1}}-1\right)^{\frac{1}{2}}\left[(4 n-5) s^{\frac{1}{n-1}}-2(n-2)\right]} .
$$

Then, taking second derivative of (3.5) with respect to the entropy and after that plugging in the solution for the charge (3.8), we get

$$
\left.\frac{\partial^{2} T}{\partial S^{2}}\right|_{Q_{c}}=\frac{\pi P_{n}\left(s^{\frac{1}{n-1}}\right)}{4 \ell(n-1)^{2} s^{\frac{2 n-3}{n-1}}\left((4 n-5) s^{\frac{1}{n-1}}+4-2 n\right)\left(s^{\frac{1}{n-1}}-1\right)}
$$

where the polynomial $P_{n}(x)$ is always cubic in $x=s^{\frac{1}{n-1}}$, and is given by

$$
P_{n}(x)=(2 n-1)(4 n-5) x^{3}+\left(-16 n^{2}+29 n-\frac{9}{2}\right) x^{2}+10 n(n-2) x-2 n(n-2) .
$$

In first few even dimensions, it has the form

$$
\begin{aligned}
& P_{2}(x)=9 x^{3}-\frac{21}{2} x^{2}, \\
& P_{3}(x)=35 x^{3}-\frac{123}{2} x^{2}+30 x-6, \\
& P_{4}(x)=77 x^{3}-\frac{289}{2} x^{2}+80 x-16 .
\end{aligned}
$$




\begin{tabular}{|c|c||c|c|c|}
\hline$D$ & $n$ & $S_{c}$ & $Q_{c}$ & $T_{c}$ \\
\hline 4 & 2 & 0.5236 & 0.1667 & 0.2599 \\
\hline 6 & 3 & 0.9058 & 0.03583 & 0.1747 \\
\hline 8 & 4 & 1.1366 & 0.007146 & 0.1391 \\
\hline
\end{tabular}

Table 1: The critical points with $k=1$ in different even dimensions.

The inflection points are identified from $P_{n}=0$. We search for strictly positive (real) solutions $x$. In four dimensions, for example, we find

$$
P_{2}=0 \quad \Rightarrow \quad x=s_{c}=\frac{7}{6}
$$

so that the critical value of the quantities obtained from (3.8) and (3.5) are

$$
\begin{aligned}
S_{c} & =\frac{\pi \ell^{2}}{6} \simeq 0.5236 \ell^{2}, \\
Q_{c} & =\frac{\ell}{6} \simeq 0.1667 \ell, \\
T_{c} & =\frac{1}{\pi \ell} \sqrt{\frac{2}{3}} \simeq \frac{0.2599}{\ell} .
\end{aligned}
$$

An asymptotically flat limit of spacetime corresponds to $\ell \rightarrow \infty$. In that case, from (3.9), the critical temperature vanishes, because the entropy parameter $s$ is just a dimensionless number obtained as a root of $P_{n}$. This means that the van del Waals-like critical behavior occurs only in an spacetime possessing a cosmological constant, as it was observed in reference [10].

From now on, we shall set the AdS radius $\ell=1$, for the sake of simplicity.

The critical points exist in higher dimensions as well, but the polynomial $P_{n}=0$ is more difficult to solve analytically when $n>2$. However, it can be checked that $P_{n}$ has the discriminant zero $(n=2)$ or negative $(n>2)$, so there is only one real root and it is always positive, which means that there is always exactly one critical point in each even dimension. We list the corresponding critical points in various even dimensions in table 1. We see that larger dimension increases the critical entropy, while it suppresses the critical charge and temperature.

The $S$-T diagrams related to the equation (3.6) for different even dimensions are shown in figure 1. It is clear that each plot possesses a boundary curve corresponding to the critical charge $Q_{c}=$ const (red dashed line), which shows an inflection point describing a second order phase transition at the critical point. The specific heat capacity is divergent there. The lines below $Q_{c}$ (represented by a black dotted line), with $Q>Q_{c}$, are monotonous curves without inflection points, which means there is no phase transition 

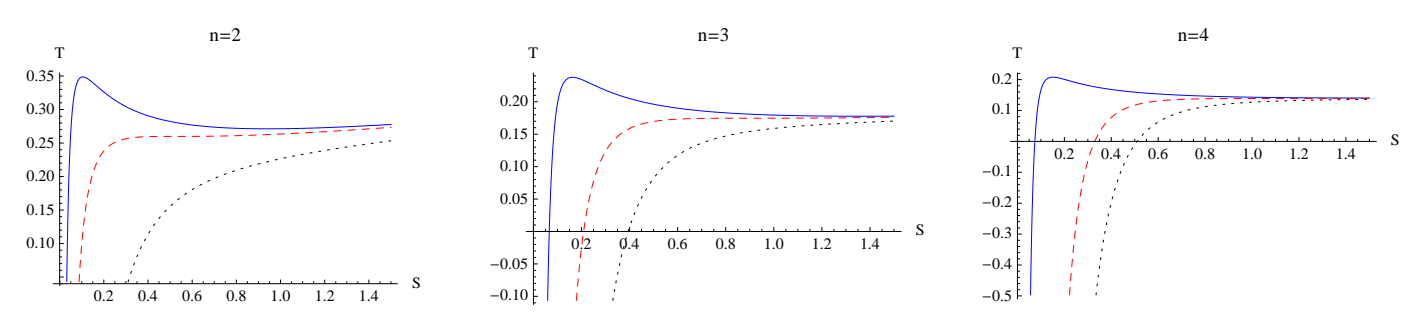

Figure 1: T-S diagram for the black hole with fixed charge in different even dimensions. In each plot, the red dashed line corresponds to the related critical charge $Q_{c}$, listed in table1. The blue line and black dotted line are related to the fixed charge which is smaller and larger than $Q_{c}$, respectively.
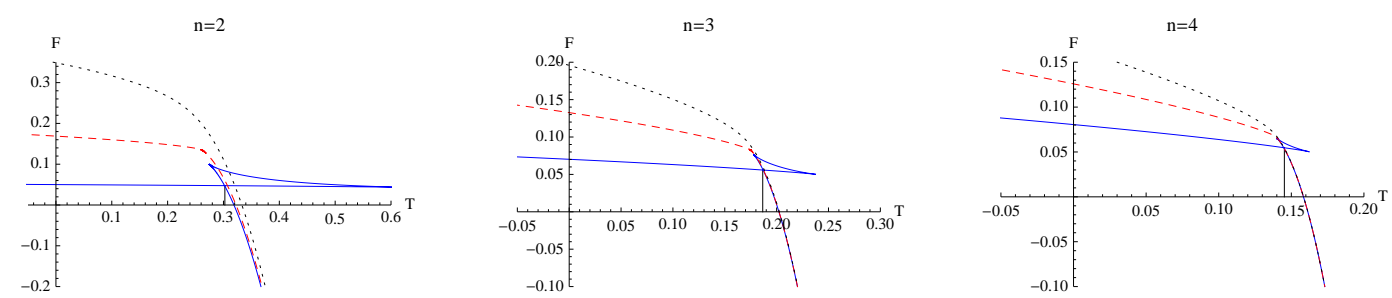

Figure 2: Free energy in terms of the temperature. The temperature of the crossing point in the blue line is the first order phase transition temperature $T_{s}$ for $Q=0.3 Q_{c}$. It shrinks into $T_{s}=T_{c}$ as $Q$ increases to $Q_{c}$, as the red dashed line shows.

in these cases. The isocharge lines above $Q_{c}$ (represented by a blue line), with $Q<Q_{c}$, are the lines which are not monotonous, and their shape is similar to the one describing a van der Waals phase transition.

More specifically, for the solid lines in each plot with fixed charge $Q<Q_{c}$, there are three black holes sharing one temperature but with different free energies to compete. There exists a critical temperature $T_{s}$ below which the smallest black hole always has the lowest free energy and is thermodynamically stable, and above which the largest black is stable. For the dashed line with $Q=Q_{c}$, the two black holes merge into one at $T_{s}=T_{c}$. For the dotted line with $Q>Q_{c}$, there is no any competition. This phenomenon is described in figure 2, where we show the relation between the temperature and free energy

$$
F=M-T S
$$

On the other hand, for a small charge with $Q=0.3 Q_{c}<Q_{c}$ (blue lines), the free energy graph shows a 'swallow tail' in the $F-T$ plane, which is typical for a first order phase transition. The horizontal coordinates of the black line in each plot denote the first order phase transition temperature $T_{s}$ with the related parameters. Furthermore, for the 
critical charge $Q_{c}$ (red dashed lines) at the inflection point where we have $T_{s}=T_{c}$, the curves show second order phase transition because equation (2) implies that the specific heat capacity $C_{Q}=\left.T \frac{\partial S}{\partial T}\right|_{Q}$ is divergent. Critical phenomena discussion from a divergence of $C_{Q}$ has also been developed in [31, 32].

Near the critical inflection point $\left(Q_{c}, S_{c}, T_{c}\right)$, we can calculate the critical exponent $\alpha$, which characterizes a behavior of the heat capacity near the critical point as

$$
C_{Q}=\left.T \frac{\partial S}{\partial T}\right|_{Q} \simeq A\left(T-T_{c}\right)^{\alpha}
$$

To determine $\alpha$, we expand the temperature (3.6) near the critical point $S_{c}$, when $Q_{c}$ is kept fixed,

$T=T\left(S_{c}, Q_{c}\right)+\left.\frac{\partial T}{\partial S}\right|_{c}\left(S-S_{c}\right)+\left.\frac{1}{2} \frac{\partial^{2} T}{\partial S^{2}}\right|_{c}\left(S-S_{c}\right)^{2}+\left.\frac{1}{6} \frac{\partial^{3} T}{\partial S^{3}}\right|_{c}\left(S-S_{c}\right)^{3}+\mathcal{O}\left(\left(S-S_{c}\right)^{4}\right)$.

Here ' $c$ ' denotes the point $\left(Q_{c}, S_{c}\right)$. The first term is $T\left(S_{c}, Q_{c}\right)=T_{c}$, while from the conditions (3.7), the second and third terms of (3.17) vanish. This yields near the critical point

$$
T-\left.T_{c} \simeq \frac{1}{6} \frac{\partial^{3} T}{\partial S^{3}}\right|_{c}\left(S-S_{c}\right)^{3} .
$$

We also calculate $\left.\frac{\partial S}{\partial T}\right|_{Q}$ expanded near the critical point by taking a partial derivative in $S$ of the expression (3.18),

$$
\left.\left.\frac{\partial T}{\partial S} \simeq \frac{1}{2} \frac{\partial^{3} T}{\partial S^{3}}\right|_{c}\left(S-S_{c}\right)^{2} \Rightarrow \frac{\partial S}{\partial T} \simeq 2 \frac{\partial^{3} T}{\partial S^{3}}\right|_{c} ^{-1}\left(S-S_{c}\right)^{-2}
$$

Thus, the heat capacity expands as

$$
C_{Q}=\left.\left.T \frac{\partial S}{\partial T}\right|_{Q} \simeq 2 T_{c} \frac{\partial^{3} T}{\partial S^{3}}\right|_{c} ^{-1}\left(S-S_{c}\right)^{-2}
$$

Now we invert the series (3.18),

$$
S-\left.S_{c} \simeq \sqrt[3]{6} \frac{\partial^{3} T}{\partial S^{3}}\right|_{c} ^{-\frac{1}{3}}\left(T-T_{c}\right)^{\frac{1}{3}},
$$

and obtain

$$
C_{Q} \simeq A\left(T-T_{c}\right)^{-\frac{2}{3}}
$$

We conclude that the heat capacity diverges at the critical point, as expected. The constant $A=\frac{1}{3} \sqrt[3]{6} T_{c}\left(\partial_{S}^{3} T\right)_{c}^{-1 / 3}$ can be evaluated explicitly by taking derivatives of (3.5). 


\begin{tabular}{|c|c||c|c|c|c|c|c|}
\hline$D$ & $n$ & $T_{s}$ & $S_{l}$ & $S_{m}$ & $S_{s}$ & $(3.23)_{L}$ & $(3.23)_{R}$ \\
\hline 4 & 2 & 0.3020 & 2.5405 & 0.4121 & 0.009865 & 0.7534 & 0.7533 \\
\hline 6 & 3 & 0.1870 & 2.8250 & 0.6703 & 0.09581 & 0.5104 & 0.5103 \\
\hline 8 & 4 & 0.1448 & 2.7920 & 0.8563 & 0.2300 & 0.3709 & 0.3708 \\
\hline
\end{tabular}

Table 2: The first order phase transition temperature with fixed $Q=0.3 Q_{c}$ and $k=1$ in different even dimensions, and the values of (3.23) which show that the Maxwell area law is satisfied. $(3.23)_{L}$ and $(3.23)_{R}$ denote the values of the left and right sides of equation (3.23).

We read-off the critical exponent $\alpha=-2 / 3$, which is independent of the dimension. This exponent agrees with the one found in the mean field theory. A general approach to obtain a critical exponent of black hole phase transition has been proposed in refs. [33, 34], where it was shown that the result does not depend on a particular black hole solution.

For the first order phase transition with $Q<Q_{c}$, the physical $T-S$ diagram should be modified by replacing the oscillating part by an isobar with $T=T_{s}$, where $T_{s}$ is the first phase transition temperature. Since, at the phase transition point, the free energy does not change, integration of the first law of thermodynamics implies $\oint S d T=0$. This leads to the Maxwell's equal area law

$$
T_{s}\left(S_{l}-S_{s}\right)=\int_{S_{s}}^{S_{l}} T d S,
$$

where $S_{l}$ and $S_{s}$ are the largest and smallest entropies of three intersection points, respectively, between the $S-T$ diagram and the related isobar $T=T_{s}$, i.e., the three solutions $\left(S_{l}, S_{m}, S_{s}\right)$ of $S$ to the equation (3.6) with $T=T_{s}$ for fixed charge. We calculated both sides of equation (3.23) with $Q=0.3 Q_{c}$, and the results are summarized in table 2 . It turns out that the Maxwell equal area law (3.23) is satisfied in considered even dimensions, with an acceptable error between analytical (left side) and numerical (right side) calculation.

So far, we have obtained that for the charged Born-Infeld black hole with the spherical horizon $(k=1)$, there exists a critical inflection point $\left(Q_{c}, S_{c}, T_{c}\right)$, near which the critical exponent matches the values in the mean field theory. The system presents a van der Waals-like phase transition in the $T$ - $S$ plane of the state equation and the Maxwell equal area law is checked to be satisfied.

In cases with non-compact horizons $(k=-1$ and $k=0)$, the state equation (3.5) 
generalizes to

$$
T=\frac{-Q^{2} s^{\frac{2-n}{n-1}}+\left((2 n-1) s^{\frac{1}{n-1}}-(2 n-2) k\right)\left(s^{\frac{1}{n-1}}-k\right)^{n-1}}{4 \pi(n-1)\left(s^{\frac{1}{n-1}}-k\right)^{n-\frac{1}{2}}}
$$

where $s=\frac{S}{\pi}+k$. Using the same analysis as above, we write the inflection point equations (3.7) and find that there are no positive solutions for $S_{c}$ and $Q_{c}$. Explicitly, in four dimensions $(n=2)$, we get analytically

$$
\begin{aligned}
S_{c} & =\frac{4 k-3}{6} \pi, \\
Q_{c} & =\frac{1}{6} \sqrt{52 k^{2}-60 k+9},
\end{aligned}
$$

so the entropy becomes negative when $k=0$ or -1 . Similar result holds in higher dimensions, as well. This means that there are no van der Waals-like phase transitions in black holes with non-compact horizons. This result that the existence of the van der Waals-like phase depends on the topology of the horizon is also valid in the extended phase space of RN AdS black holes [10].

\section{Odd-dimensional charged black holes and thermal phase transitions}

In odd dimensions $D=2 n-1$, the charged Chern-Simons AdS black hole (2.13) with $G=1$ has the form [17],

$$
f^{2}(r)=k+\frac{r^{2}}{\ell^{2}}-\left(2 M+1-\frac{Q^{2}}{(D-3) r^{D-3}}\right)^{\frac{1}{n-1}} .
$$

In three dimensions $(n=2)$, this solution is the BTZ black hole [27].

The Hawking temperature for an arbitrary $n$ reads

$$
T=\frac{r_{+}}{2 \pi \ell^{2}}-\frac{Q^{2}\left(k+\frac{r_{+}^{2}}{\ell^{2}}\right)^{2-n}}{4 \pi(n-1) r_{+}^{2 n-3}}
$$

and the entropy is given in the parametric form,

$$
S=4 \pi(n-1) r_{+} \int_{0}^{1} d u\left(k+u^{2} \frac{r_{+}^{2}}{\ell^{2}}\right)^{n-2} .
$$


We apply the same strategy as in the last section. Since the equation $S\left(r_{+}\right)$cannot be inverted in a simple way to obtain $r_{+}(S)$, it is convenient to calculate derivatives in eqs.(3.7) as

$$
\left.\frac{\partial T}{\partial S}\right|_{Q}=\frac{\left.\frac{\partial T}{\partial r_{+}}\right|_{Q}}{\frac{d S}{d r_{+}}}
$$

We find (with $\ell=1$ )

$$
\begin{aligned}
\frac{d S}{d r_{+}} & =4 \pi(n-1) \int_{0}^{1} d u\left[k+(2 n-3) u^{2} r_{+}^{2}\right]\left(k+u^{2} r_{+}^{2}\right)^{n-3} \\
& =4 \pi(n-1)\left(k+r_{+}^{2}\right)^{n-2}
\end{aligned}
$$

as well as

$$
\left.\frac{\partial T}{\partial r_{+}}\right|_{Q}=\frac{1}{2 \pi}+\frac{Q^{2}\left[(2 n-3) k+(4 n-7) r_{+}^{2}\right]\left(k+r_{+}^{2}\right)^{1-n}}{4 \pi(n-1) r_{+}^{2 n-2}}
$$

what implies vanishing $\partial T /\left.\partial S\right|_{Q}$ for

$$
Q_{c}^{2}=-\frac{2(n-1) r_{+}^{2 n-2}}{\left[(2 n-3) k+(4 n-7) r_{+}^{2}\right]\left(k+r_{+}^{2}\right)^{1-n}}, \quad r_{+} \neq-k .
$$

It is clear that $Q_{c}^{2}$ can be positive only for hyperbolic black holes, so the planar ones are ruled out of having a phase transition of the considered type. Setting $k=-1$ and using again the same method to calculate the second derivative of the entropy, we obtain that $\left.\frac{\partial^{2} T}{\partial S^{2}}\right|_{Q}$ replaced from (4.7)) vanishes when

$$
\left(8 n^{2}-26 n+21\right) r_{+}^{4}-\left(8 n^{2}-25 n+20\right) r_{+}^{2}+2 n^{2}-5 n+3=0 .
$$

The discriminant of this quadratic polynomial in $r_{+}^{2}$ is always negative when $n>2$, so there is no a real solution for $r_{+}$and, therefore, there is no a critical point. When $n=2$, the root is $r_{+}=1$, but this point is not allowed because of the inequality in (4.7). An independent analysis in three dimensions shows that $Q_{c}^{2}$ becomes negative, so again there is no a critical point when $n=2$.

We conclude that, in all odd dimensions, Chern-Simons AdS topological black holes, including the BTZ black hole, do not admit a van der Waals-like phase transition for any geometry of the horizon.

\section{Conclusions and discussion}

We analyzed the thermal phase transitions of charged dimensionally continued black holes in the $T$-S plane. In even dimensions, we found that the critical inflection point $\left(Q_{c}, S_{c}, T_{c}\right)$ 
can only exist if the black hole has a spherical horizon (with $k=1$ ), and not if it has a non-compact geometry $(k=0$ or $k=-1$.) For $k=1$, near the critical point, the critical exponent in the specific heat capacity is always $-2 / 3$ in any even dimension and this values agree with that of the mean field theory. Moreover, we found that the system goes through a van der Waals-like phase transition. When the charge is smaller than the critical charge, it undergoes the first order phase transition at $T=T_{s}$, below which the smallest black hole always has the lowest free energy and is thermodynamically stable while, above it, the largest black hole is thermodynamically stable. The Maxwell equal area law has been checked to be satisfied at the first order phase transition point in first few even dimensions.

We did not observe a van der Waals-like phase transition in odd-dimensional black holes in the charged dimensionally continued gravity.

It is important to mention that, at first sight, two families of DCG, Chern-Simons AdS and Born-Infeld AdS gravity, seem similar, but they are intrinsically different. First, Chern-Simons gravity comes from a Chern-Simons form, whose exterior derivative is a topological invariant. In contrast, Born-Infeld gravity does not have a geometric origin. Furthermore, in comparison to all Lovelock AdS actions, Chern-Simons AdS features a symmetry enhancement from local Lorentz to local AdS group, what drastically changes its dynamic structure and a number of local degrees of freedom. While all generic Lovelock gravities have the same number of degrees of freedom as General Relativity [28], even a Pure Lovelock [29] which does not posses the linear in curvature term, this is no longer true for Chern-Simons gravity [30]. From this point of view, it is not surprising that its phase space structure is drastically different than the one of Born-Infeld.

It is worthwhile noticing that, with the development of holographic duality, it was observed in [35] that there also exists the van der Waals-like phase transition in the entanglement entropy-temperature $\left(S_{E}-T\right)$ diagram by studying the holographic entanglement entropy (see [36] for review) in a finite volume quantum system dual to a charged AdS black hole with spherical geometry. More related studies in [24, 37, 38, 39, 40] indicate that similar to thermal entropy, the entanglement entropy also presents the van der Waals-like phase behavior. Thus, it would be very interesting and important to explore the holographic entanglement entropy of the charged dimensional continued black holes, especially the one with spherical horizon in even dimensions, in which the van der Waalslike phase transition in $S-T$ has been observed. We will address this question in the near future. 


\section{Acknowledgments}

This work was funded by the Chilean FONDECYT Grant No.3150006 and the VRIEAPUCV grant No.039.345/2016.

\section{References}

[1] A. Chamblin, R. Emparan, C. V. Johnson and R. C. Myers, "Charged AdS black holes and catastrophic holography," Phys. Rev. D 60, 064018 (1999) hep-th/9902170.

[2] A. Chamblin, R. Emparan, C. V. Johnson and R. C. Myers, "Holography, thermodynamics and fluctuations of charged AdS black holes," Phys. Rev. D 60, 104026 (1999) hep-th/9904197.

[3] D. Kastor, S. Ray and J. Traschen, "Enthalpy and the Mechanics of AdS Black Holes," Class. Quant. Grav. 26, 195011 (2009) arXiv:0904.2765 [hep-th]].

[4] M. Cvetic, G. W. Gibbons, D. Kubiznak and C. N. Pope, "Black Hole Enthalpy and an Entropy Inequality for the Thermodynamic Volume," Phys. Rev. D 84, 024037 (2011) arXiv:1012.2888 [hep-th]].

[5] B. P. Dolan, "Pressure and volume in the first law of black hole thermodynamics," Class. Quant. Grav. 28, 235017 (2011) [arXiv:1106.6260 [gr-qc]].

[6] B. P. Dolan, "Compressibility of rotating black holes," Phys. Rev. D 84, 127503 (2011) [arXiv:1109.0198 [gr-qc]].

[7] D. A. Rasheed, "Nonlinear electrodynamics: Zeroth and first laws of black hole mechanics," hep-th/9702087.

[8] N. Breton, "Smarr's formula for black holes with non-linear electrodynamics," Gen. Rel. Grav. 37, 643 (2005) gr-qc/0405116.

[9] W. Yi-Huan, "Energy and first law of thermodynamics for Born-Infeld AdS black hole," Chin. Phys. B 19, 090404 (2010).

[10] D. Kubiznak and R. B. Mann, "P-V criticality of charged AdS black holes," JHEP 1207, 033 (2012) [arXiv:1205.0559 [hep-th]].

[11] D. C. Zou, S. J. Zhang and B. Wang, "Critical behavior of Born-Infeld AdS black holes in the extended phase space thermodynamics," Phys. Rev. D 89, 044002 (2014) arXiv:1311.7299 [hep-th]]. 
[12] J. X. Mo and W. B. Liu, " $P-V$ criticality of topological black holes in LovelockBorn-Infeld gravity," Eur. Phys. J. C 74, 2836 (2014) [arXiv:1401.0785 [gr-qc]].

[13] R. A. Hennigar, W. G. Brenna and R. B. Mann, " $P-V$ criticality in quasitopological gravity," JHEP 1507, 077 (2015) [arXiv:1505.05517 [hep-th]].

[14] J. Xu, L. M. Cao and Y. P. Hu, "P-V criticality in the extended phase space of black holes in massive gravity," Phys. Rev. D 91, 124033 (2015) [arXiv:1506.03578 [gr-qc]].

[15] S. H. Hendi, A. Sheykhi, S. Panahiyan and B. Eslam Panah, "Phase transition and thermodynamic geometry of Einstein-Maxwell-dilaton black holes," Phys. Rev. D 92, 064028 (2015) arXiv:1509.08593 [hep-th]].

[16] B. R. Majhi and S. Samanta, "P-V criticality of AdS black holes in a general framework," arXiv:1609.06224 [gr-qc].

[17] M. Bañados, C. Teitelboim and J. Zanelli, "Dimensionally continued black holes," Phys. Rev. 49, 975 (1994) gr-qc/9307033.

[18] J. Crisostomo, R. Troncoso and J. Zanelli, "Black hole scan," Phys. Rev. D62, 084013 (2000) hep-th/0003271.

[19] A. H. Chamseddine, "Topological gauge theory of gravity in five dimensions and all odd dimensions," Phys. Lett. B233, 291 (1989).

[20] R. G. Cai and K. S. Soh, "Topological black holes in the dimensionally continued gravity," Phys. Rev. D 59, 044013 (1999) gr-qc/9808067].

[21] J. P. Muniain and D. D. Piriz, "Critical behavior of dimensionally continued black holes," Phys. Rev. D 53, 816 (1996) gr-qc/9502029.

[22] O. Miskovic and R. Olea, "Counterterms in Dimensionally Continued AdS Gravity," JHEP 0710, 028 (2007) arXiv:0706.4460 [hep-th]].

[23] E. Spallucci and A. Smailagic, "Maxwell's equal area law for charged anti-de Sitter black holes," Phys. Lett. B 723, 436 (2013) [arXiv:1305.3379 [hep-th]].

[24] X. X. Zeng, H. Zhang and L. F. Li, "Phase transition of holographic entanglement entropy in massive gravity," Phys. Lett. B 756, 170 (2016) [arXiv:1511.00383 [gr-qc]].

[25] D. Lovelock, "The Einstein tensor and its generalizations", J. Math. Phys. 12, 498 (1971). 
[26] C. Lanczos, "The four-dimensionality of space and the Einstein tensor", J. Math. Phys. 13, 874 (1972).

[27] M. Banados, C. Teitelboim and J. Zanelli, "The Black hole in three-dimensional space-time," Phys. Rev. Lett. 69, 1849 (1992) hep-th/9204099.

[28] C. Teitelboim, J. Zanelli, "Dimensionally continued topological gravitation theory in Hamiltonian form," Class. Quantum Grav. 4 L125 (1987).

[29] N. Dadhich, R. Durka, N. Merino and O. Miskovic, "Dynamical structure of Pure Lovelock gravity," Phys. Rev. D 93, 064009 (2016)

[30] M. Bañados, L. J. Garay and M. Henneaux, "The Dynamical structure of higher dimensional Chern-Simons theory," Nucl. Phys. B 476, 611 (1996) [hep-th/9605159]. arXiv:1511.02541 [hep-th]].

[31] R. Banerjee and D. Roychowdhury, "Critical phenomena in Born-Infeld AdS black holes," Phys. Rev. D 85, 044040 (2012) [arXiv:1111.0147 [gr-qc]].

[32] R. Banerjee and D. Roychowdhury, "Critical behavior of Born Infeld AdS black holes in higher dimensions," Phys. Rev. D 85, 104043 (2012) [arXiv:1203.0118 [gr-qc]].

[33] A. Mandal, S. Samanta and B. R. Majhi, "Phase transition and critical phenomena of black holes: A general approach," Phys. Rev. D 94, no. 6, 064069 (2016) doi:10.1103/PhysRevD.94.064069 [arXiv:1608.04176] [gr-qc]].

[34] R. Banerjee, B. R. Majhi and S. Samanta, "Thermogeometric phase transition in a unified framework," arXiv:1611.06701 [gr-qc].

[35] C. V. Johnson, "Large N Phase Transitions, Finite Volume, and Entanglement Entropy," JHEP 1403, 047 (2014) [arXiv:1306.4955 [hep-th]].

[36] S. Ryu and T. Takayanagi, "Aspects of Holographic Entanglement Entropy," JHEP 0608, 045 (2006) hep-th/0605073.

[37] E. Caceres, P. H. Nguyen and J. F. Pedraza, "Holographic entanglement entropy and the extended phase structure of STU black holes," JHEP 1509, 184 (2015) arXiv:1507.06069 [hep-th]].

[38] A. Dey, S. Mahapatra and T. Sarkar, "Thermodynamics and Entanglement Entropy with Weyl Corrections," Phys. Rev. D 94, 026006 (2016) arXiv:1512.07117 [hep-th]]. 
[39] Y. Sun, H. Xu and L. Zhao, "Thermodynamics and holographic entanglement entropy for spherical black holes in 5D Gauss-Bonnet gravity," JHEP 1609, 060 (2016) arXiv:1606.06531 [gr-qc]].

[40] S. He, L. F. Li and X. X. Zeng, "Holographic van der Waals-like phase transition in the Gauss-Bonnet gravity," arXiv:1608.04208 [hep-th]. 